\title{
Neue Prinzipien in der Therapie affektiver Störungen
}

Depressionen gehören zu den großen Volkskrankheiten und nehmen vor allem in Industriestaaten hinsichtlich ihrer Häufigkeit weiterhin zu. Ferner sind sie eine der wesentlichen Ursachen für Frühverrentung. Mittlerweile steht eine Reihe von Antidepressiva zur Behandlung depressiver Erkrankungen zur Verfügung, welche zusammen mit weiteren Therapieverfahren (z. B. Psychotherapie, Hirnstimulationsverfahren, Wachtherapie etc.) durchaus erfolgreich in der Behandlung depressiver Patienten eingesetzt werden. Dennoch bedarf es weiterer Forschungsanstrengungen hinsichtlich der Neuentwicklung von Antidepressiva, da ca. 30\% der depressiven Patienten nur unzureichend auf eine Monotherapie ansprechen. Insbesondere der langsame Wirkungseintritt von bis zu mehreren Wochen stellt ein großes Problem in der Therapie depressiver Störungen dar. Hinsichtlich des Wirkmechanismus von Antidepressiva ist davon auszugehen, dass die meisten auf dem Markt befindlichen Präparate eine Wiederaufnahme der Neurotransmitter Serotonin und/oder Noradrenalin in die präsynaptische Nervenendigung durch Blockade der entsprechenden Transporter hemmen. Hierdurch kommt es zu einer vermehrten Verfügbarkeit der entsprechenden Neurotransmitter im synaptischen Spalt mit nachfolgender Wirkung auf entsprechende postsynaptische Rezeptoren. Mittlerweile werden jedoch auch andere Mechanismen als relevant für die Wirkung von Antidepressiva diskutiert. Diese schließen eine Abschwächung der Überaktivität des Hypothalamus-Hypophysen-Nebennierenrinden-Systems, welche bei depressiven Patienten häufig beobachtet wird sowie Wechselwirkungen mit postsynaptischen Signaltransduktionskaskaden ein. Attraktiv erscheint auch die Hypothese, dass Antidepressiva die Expression von neurotrophen Faktoren steigern können. Schließlich konnte unsere Arbeitsgruppe zeigen, dass Antidepressiva ligandengesteuerte Ionenkanäle modulieren können. Wesentlich für die Wirkung von Antidepressiva in der Zellmembran sind sog. Lipid Rafts. Lipid Rafts sind Mikrodomänen innerhalb der Zell- membran, welche reich an Cholesterol und Sphingolipiden sind. Wir konnten zeigen, dass Antidepressiva, welche in der Lage sind, ligandengesteuerte lonenkanäle zu modulieren, sich vorzugsweise in diesen Lipid Rafts anreichern. Andererseits werden Benzodiazepine vorzugsweise in non-raft Fraktionen aufgenommen. Die genaue Lokalisation von Neurotransmitter-Rezeptoruntereinheiten bzw. der Anreicherung von Psychopharmaka innerhalb entsprechender Mikrodomänen in der Zellmembran spielt somit eine wesentliche Rolle für die pharmakologische Wirkung von Psychopharmaka auf subzellulärer Ebene.

In aktuellen Forschungsprojekten untersuchen wir die Wirkung von Antidepressiva auf Gliazellen. Gliazellen sind geeignet, auch die Funktion neuronaler Synapsen zu modulieren. Wir konnten zeigen, dass Antidepressiva bestimmte gliale Proteine hochregulieren, die ihrerseits das Aussprossen von Fortsätzen in Astrozyten begünstigen. Insofern beeinflussen Antidepressiva über die gliale Morphologie die Funktion neuronaler Synapsen. Ferner konnten wir zeigen, dass Antidepressiva MAP-Kinasen in Gliazellen aktivieren können, während die Aktivität dieser Kinasen in Neuronen reduziert wird. Solche Muster finden sich vor allem während der Gehirnreifung und deuten darauf hin, dass Antidepressiva gewissermaßen zu einer Art Reset-Mechanismus führen, welcher für ihre Wirkung mit verantwortlich sein könnte.

Hinsichtlich der Therapie von Angstsymptomen kommt nach wie vor den Benzodiazepinen ein beträchtlicher Stellenwert zu. Allerdings weisen Benzodiazepine ein typisches Nebenwirkungsprofil auf, welches neben der Sedierung bei akuter Gabe vor allem bei mittel- bis längerfristiger Therapiedauer Toleranzentwicklung sowie Absetzprobleme bis hin zur Abhängigkeit beinhaltet. Daher besteht Bedarf an rasch wirksamen Anxiolytika, welche diese $\mathrm{Ne}$ benwirkungen vermissen lassen. Wir sind der Frage nachgegangen, inwieweit Ligan- den des sog. Translokator-Proteins 18 kDa (TSPO) geeignet sind, eine solche Klasse von neuartigen Anxiolytika darzustellen. Wir konnten zeigen, dass der TSPO-Ligand XBD173 die GABA-vermittelte Neurotransmission über eine Induktion der Neurosteroidogenese verstärken kann. Ferner wirkt dieser TSPO-Ligand in verschiedenen Tiermodellen anxiolytisch. In einem translationalen Ansatz konnten wir anxiolytische Effekte für XBD173 im CholecystokininTetrapeptid (CCK4)-Modell sowohl bei der Ratte als auch bei gesunden Probanden in einer Proof of Concept-Studie nachweisen. Dabei erwies sich XBD173 sowie Alprazolam der Placebobedingung hinsichtlich der Reduktion der CCK4-induzierten Angst überlegen. Im Unterschied zu Alprazolam fand sich keine Sedierung und keine Absetzphänomene nach einwöchiger Gabe von XBD173 während bei Alprazolam bereits nach einer Woche Gabe bei fast $60 \%$ der Probanden Absetzphänomene zu beobachten waren. In Nachfolgestudien konnten wir zeigen, dass die Wirkung von XBD173 auch von der genetischen Ausstattung abhängt. So ist der Alanin/Threonin-Polymorphismus an der Position 147 des TSPO-Proteins entscheidend für die Bindungsaktivität von TSPO-Liganden. TSPO-Liganden können aber auch eine Rolle für die Behandlung neurodegenerativer Erkrankungen sowohl im peripheren als auch im zentralen Nervensystem spielen, indem sie zu einer Reduktion der Mikrogliaaktivierung beitragen.

Es bleibt zu hoffen, dass angesichts derzeitig stagnierender Neuentwicklungen im Bereich der Neuropsychopharmakologie interessante neuartige Zielstrukturen, z. B. TSPO, identifiziert werden, die zur Entwicklung neuer Psychopharmaka mit günstigerem Nebenwirkungsprofil beitragen.

\section{Interessenkonflikte}

Der Autor hat Beratungshonorare sowie Kostenvergütungen für die Teilnahme an Kongressen und Honorare für Vorträge erhalten von den Firmen Novartis, Servier, Grünenthal. 
Autor

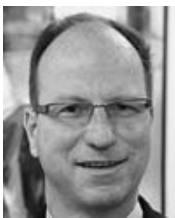

Rainer Rupprecht Klinik für Psychiatrie und Psychotherapie der Universität Regensburg

\section{Korrespondenzadresse}

\section{Prof. Dr. Rainer Rupprecht}

Direktor

Klinik für Psychiatrie und

Psychotherapie

Universität Regensburg am Bezirksklinikum

Universitätsstraße 84

93053 Regensburg

Rainer.Rupprecht@medbo.de

\section{Literatur}

[1] Di Benedetto B, Rupprecht R, Czéh B. Talking to the synapse: How antidepressants can target glial cells to reshape brain circuits. Current Drug Targets 2013; 14: 1329-1335

[2] Di Benedetto B, Rupprecht R. Targeting glia cells: novel perspectives for the treatment of neuropsychiatric diseases. Current Neuropharmacology 2013; 11: 171-185

[3] Rupprecht R, Papadopoulos V, Rammes G et al. Translocator protein $(18 \mathrm{kDa})$ as a therapeutic target for neurological and psychiatric disorders. Nature Reviews Drug Discovery 2010; 9: 971-988

[4] Nothdurfter C, Tanasic, S. Modulation of ligand gated ion channels as a novel pharmacological principle. Pharmacopsychiatry 2011; 44: 527-534

[5] Nothdurfter C, Rammes G, Baghai TC et al. TSPO (18 kDa) as a target for novel anxiolytics with a favourable side effect profile. Journal of Neuroendocrinology 2012; 24: 82-92

[6] Di Benedetto B, Radecke J, Schmidt MV et al. Acute antidepressant treatment differently modulates ERK/MAPK activation in neurons and astrocytes of the adult mouse prefrontal cortex. Neuroscience 2013; 232: 161-168
7] Leicht G, Mulert C, Eser D et al. Benzodiazepines counteract rostral anterior cingulate cortex activation induced by CCK-4 in humans. Biological Psychiatry 2013; 73: 337 344

[8] Karlstetter M, Nothdurfter C, Aslanidis A et al. Rupprecht R* Translocator protein (18 kDa) (TSPO) is expressed on reactive retinal microglia and modulates microglial inflammation and phagocytosis. Journal of Neuroinflammation 2014; 11: 3. doi:10.1186/17422094-11-3

[9] Di Benedetto B, Malik V, Begum S et al. Fluoxetine requires the endfeet protein aquaporin-4 to enhance plasticity of astrocyte processes. Frontiers in Cellular Neuroscience 2016; 10: 8. doi:10.3389/fncel.2016. 00008. eCollection 2016

[10] Rupprecht R, Rammes G, Eser D et al. Translocator Protein $(18 \mathrm{kDa})$ as target for anxiolytics without benzodiazepine-like side effects. Science 2009; 325: 490-493

[11] Eisensamer B, Uhr M, Meyr S et al. Antidepressants and antipsychotic drugs co-localize with serotonin type $3\left(5-\mathrm{HT}_{3}\right)$ receptors in raft-like domains. Journal of Neuroscience 2005; 25: 10198-10206 